\title{
Conserved and nonconserved functions of the yeast and human TATA-binding proteins
}

\author{
Brendan P. Cormack, ${ }^{1}$ Michel Strubin, ${ }^{2}$ Laurie A. Stargell, and Kevin Struhl ${ }^{3}$ \\ Department of Biological Chemistry and Molecular Pharmacology, Harvard Medical School, \\ Boston, Massachusetts 02115 USA
}

\begin{abstract}
Although the TATA-binding protein (TBP) is highly conserved throughout the eukaryotic kingdom, human TBP cannot functionally replace yeast TBP for cell viability. To investigate the basis of this species specificity, we examine the in vivo transcriptional activity of human TBP at different classes of yeast promoters. Consistent with previous results, analysis of yeast/human hybrid TBPs indicates that growth defects are not correlated with the ability to promote TATA-dependent polymerase II (Pol II) transcription or to respond to acidic activator proteins. Human TBP partially complements the growth defects of a yeast TBP mutant with altered TATA element-binding specificity, suggesting that it carries out sufficient Pol II function to support viability. However, human TBP does not complement the defects of yeast TBP mutants that are specifically defective in transcription by RNA polymerase III. Three independently isolated derivatives of human TBP that permit yeast cell growth replace arginine 231 with lysine; the corresponding amino acid in yeast TBP (lysine 133) has been implicated in RNA polymerase III transcription. Transcriptional analysis indicates that human TBP functions poorly at promoters recognized by RNA polymerases I and III and at RNA Pol II promoters lacking a conventional TATA element. These observations suggest that species specificity of TBP primarily reflects evolutionarily diverged interactions with TBP-associated factors (TAFs) that are necessary for recruitment to promoters lacking TATA elements.
\end{abstract}

[Key Words: TATA-binding protein; TAFs; transcription; yeast]

Received March 1, 1994; revised version accepted April 22, 1994.

The TATA-binding protein (TBP), the central initiation factor for transcription by all three nuclear RNA polymerases (Comai et al. 1992; Cormack and Struhl 1992; Schultz et al. 1992; White et al. 1992), is highly conserved throughout the eukaryotic kingdom. The carboxy-terminal core domain (180 amino acids), which is sufficient for TATA element binding (Horikoshi et al. 1990 ) and for all essential functions in yeast (Cormack et al. 1991; Gill and Tjian 1991; Poon et al. 1991; Zhou et al. 1991), is $80 \%$ identical between yeast and human. The cocrystal structures of yeast and Arabidopsis TBPs bound to the TATA element are extremely similar (J.L. Kim et al. 1993; Y. Kim et al. 1993). This remarkable conservation is reflected in the similar biochemical properties of TBPs from different species. In vitro, yeast TBP (yTBP) and human TBP (hTBP) can functionally replace one another for basal RNA polymerase II (Pol II) transcription (Buratowski et al. 1988; Cavallini et al. 1988; Flanagan et al. 1990), and they display nearly iden-

Present addresses: 'Department of Microbiology and Immunology, Stanford University, Stanford, California 94305 USA; ${ }^{2}$ Department Microbiologie, CMU, 1211 Geneva 4, Switzerland.

${ }^{3}$ Corresponding author. tical DNA sequence requirements for TATA elements (Wobbe and Struhl 1990).

Despite the high degree of structural and functional conservation, TBP does exhibit species-specific behavior in vivo. In particular, hTBP cannot functionally replace yTBP for cell viability, indicating that human TBP cannot carry out at least one essential function of yTBP (Cormack et al. 1991; Gill and Tiian 1991). This failure to support cell growth is not attributable to the divergent amino-terminal regions but to multiple amino acid differences in the carboxy-terminal core domain /Cormack et al. 1991; Gill and Tiian 1991). Another example of species specificity is the inability of yTBP to mediate a transcriptional response to retinoic acid in embryo carcinoma cells (Berkenstam et al. 1992). In this case, the functional difference maps to a short region of TBP, and it may reflect the inability of yTBP to interact with an E1A-like activity that is specific to embryo carcinoma cells (Keaveney et al. 1993).

Why is hTBP unable to support yeast cell growth? As suggested by initial experiments in vitro (Peterson et al. 1990; Pugh and Tiian 1990), one possibility is that hTBP and $y$ TBP may differ in their ability to form a functional TFIID complex that is necessary for responding to tran- 
scriptional activators. However, this view has not been supported by subsequent experiments in vitro and in vivo. First, a mutant hTBP derivative with altered DNAbinding specificity is responsive to acidic activators in yeast cells (Strubin and Struhl 1992). Second, in different in vitro studies, human and yeast TBPs respond to acidic activator proteins in both yeast and human transcription systems (Kelleher et al. 1992). Third, the nonresponsiveness of yTBP to retinoic acid induction is specific to embryo carcinoma cells and does not reflect a general defect in transcriptional activation (Keaveney et al. 1993).

These observations do not address the possibility that species-specificity might reflect defects in specific polymerase II (Pol II) functions of TBP. For example, hTBP might not function at promoters that lack conventional TATA sequences or that require gene-specific transcription factors lacking acidic activation domains. What also must be considered is that TBP is a component of the SLl (Comai et al. 1992) and TFIIIB complexes (Kassavetis et al. 1992; Lobo et al. 1992; Taggart et al. 1992), which are respectively involved in transcriptional initiation by Pol I and Pol III. In principle, therefore, the failure of hTBP to support yeast cell viability could result from a defect in transcription by any of the three RNA polymerases.

In this paper we address this issue by isolating a mutant hTBP that permits yeast cell growth, and by examining directly the transcriptional phenotype of hTBP in vivo. To circumvent the problems associated with inviability, transcriptional analysis of hTBP was carried out in a strain that also contained a temperature-sensitive derivative of yTBP. Specific Pol II and Pol III functions were also examined with the use of altered DNA-binding specificity (Strubin and Struhl 1992) and Pol III-specific mutations (Cormack and Struhl 1993) in yTBP. We show that the inability of hTBP to support cell growth in yeast does not correlate with its ability to carry out TATAdependent transcription but, rather, reflects functional defects at Pol I, Pol III, and TATA-less Pol II promoters.

\section{Results \\ Partially functional hTBP-yTBP chimeras show no obvious defect in Pol II transcription}

Previously, we used the plasmid shuffle assay to determine the function of chimeric hTBP-yTBP molecules (Cormack et al. 1991). In this assay a TRP1-marked plasmid containing the TBP derivative to be tested is introduced into yeast strain BY $\Delta 2$, which is deleted for the endogenous TBP gene and contains a URA3-marked plasmid carrying the wild-type $T B P$ gene. The ability of the TBP derivative to support cell growth is assayed by plating the resulting strains in medium containing 5-fluoro-orotic acid (5-FOA), a compound that is selectively toxic to cells containing the URA3-marked plasmid. Figure 1A shows four hTBP-yTBP hybrid proteins that support cell growth to varying extents.

To investigate whether there was a defect in Pol II transcription that corresponded with the poor growth of strains containing these yeast-human TBP hybrid proteins, we measured HIS3 and DED1 mRNA levels (Fig. 1B). Wild-type levels of the HIS $3+1$ and the DED1 transcript were observed in all cases. Surprisingly, the level of the HIS $3+13$ transcript actually increased in strains with the subfunctional TBP molecules, with the extent of increased +13 transcription being inversely proportional to the health of the cell.

Although this increase in +13 transcription could result from specific functional differences among the TBP molecules, we suspected that this effect might be attrib-

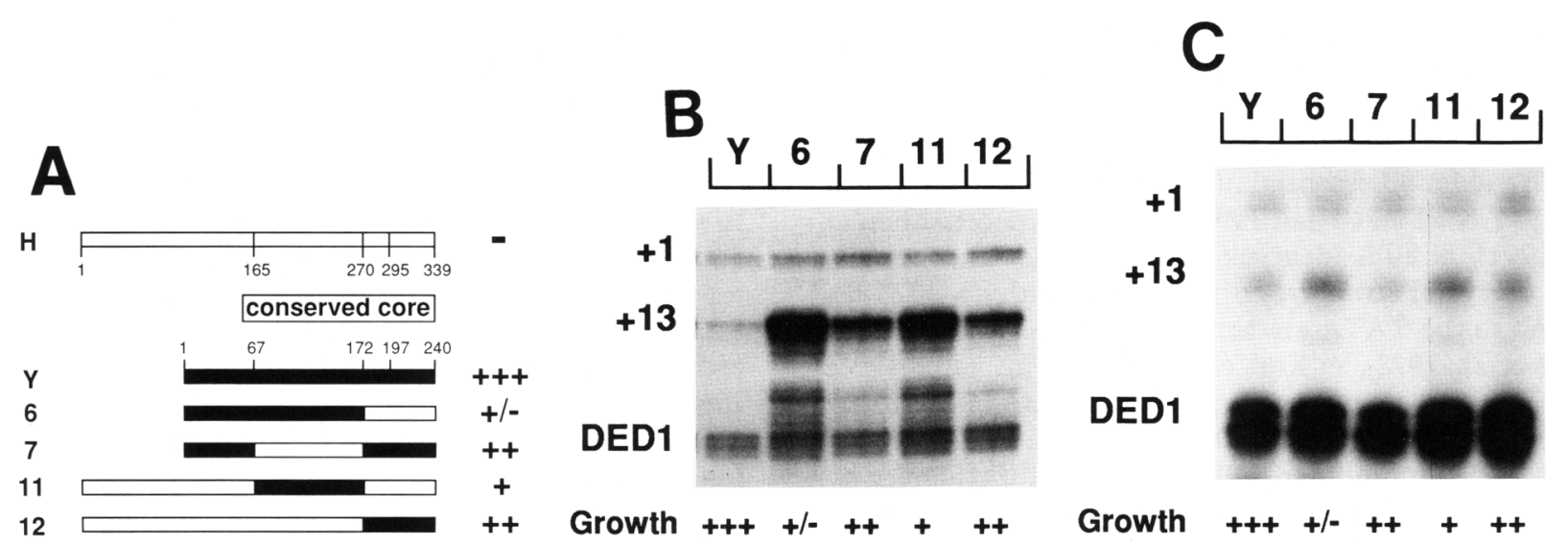

Figure 1. Transcriptional analysis of strains containing yTBP/hTBP hybrids. $(A)$ For each hybrid TBP, the portions derived from yTBP (solid) and hTBP (open) are indicated along with the positions of the hybrid junctions and the location of the highly conserved core domain. The relative abilities of these TBP derivatives to support cell growth (defined by colony sizes; see Cormack et al. 1991) are also indicated. $(B)$ Analysis of HIS3 $(+1$ and +13$)$ and DED1 RNAs in wild-type (i.e., GCN4) strains containing the indicated derivatives as the sole source of TBP. The labeled $D E D 1$ probe was diluted 10 -fold with unlabeled oligonucleotide to reduce the specific activity. (C) Analysis of $H I S 3(+1$ and +13$)$ and DED1 RNAs in isogenic gcn4 deletion strains containing the indicated derivatives as the sole source of TBP. The DED1 probe was not diluted. 
utable to induction of the transcriptional activator GCN4 under conditions of suboptimal growth. GCN4 selectively increases the +13 transcript (Struhl 1986), and GCN4 protein levels increase upon amino acid or purine limitation (Hinnebusch 1984; Thireos et al. 1984; Mosch et al. 1991; Rolfes and Hinnebusch 1993). In accord with this hypothesis, elevated levels of the +13 transcript are almost completely eliminated in gcn 4 deletion strains containing the same hybrid TBP molecules (Fig. 1C). The residual increase observed in strains containing the least functional TBP hybrids may reflect activation by YAP1 and/or YAP2, proteins that are involved in stress responses and bind weakly to the GCN4 site in the HIS3 promoter (Moye-Rowley et al. 1989; Schnell et al. 1992; Bossier et al. 1993). These results strongly suggest that the hTBP-yTBP hybrids efficiently promote basal and GCN4-activated transcription. The physiological mechanism by which suboptimal TBP molecules cause GCN4 induction is unknown, but it probably does not arise from amino acid or purine limitation because the experiments are carried out in complete growth medium.

\section{Isolation of a hTBP mutant that supports cell growth in yeast}

hTBP-yTBP hybrid 17, which contains hTBP residues 1-295 fused to amino acids 198-240 of yTBP, permits only very slow growth (Cormack et al. 1991). To obtain derivatives with improved TBP function, five independent colonies carrying hybrid 17 as the only copy of TBP were individually grown to saturation in liquid cultures, and $10^{7}$ cells from each culture were plated on YPD plates. Plasmids carrying the chimeric TBP were rescued from fast growing colonies and then retested for their ability to support cell growth. Of five such plasmids, three independent isolates conferred faster growth than hybrid 17.

DNA sequencing of the TBP-coding region revealed that all three derivatives contain the same AGA to AAA

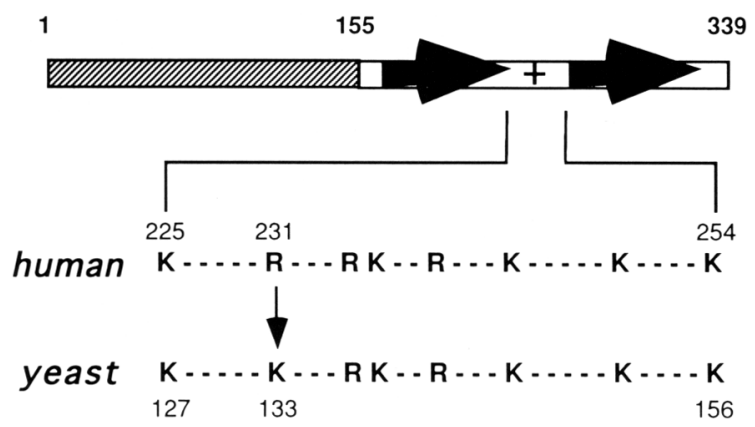

Figure 2. A derivative of hTBP that supports yeast cell growth. hTBP (339 residues) contains a divergent amino-terminal region (hatched), basic repeat $(+)$, and two direct repeats (arrows). Shown below is an expanded view of the basic repeats of yTBP (residues 127-156) and hTBP (residues 225-254), with the basic residues indicated. In the hTBP derivative that supports cell growth, arginine 231 is replaced by lysine, the corresponding residue that occurs in YTBP (K133).

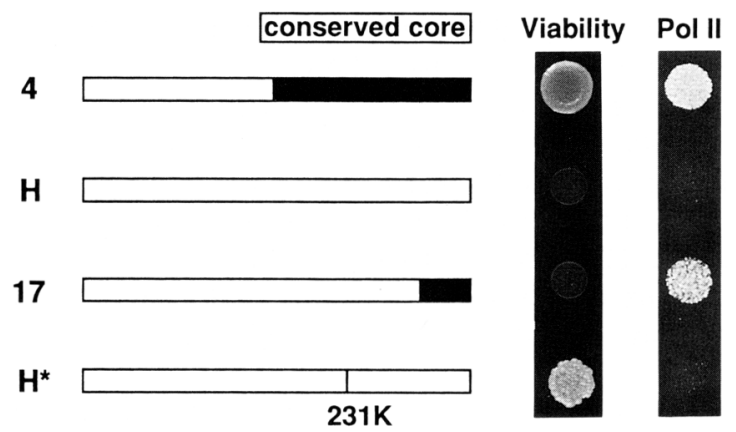

Figure 3. Cell viability is not correlated with the efficiency of supporting GCN4- and TATA-dependent Pol II transcription. Cell viability was examined by growth in the presence of 5-FOA after plasmid shuffling. The Pol II assay was performed by introducing altered specificity derivatives of the various TBPs into a strain containing a TGTAAA sequence downstream of a GCN4-binding site and examining the resulting cells for growth in medium containing $5 \mathrm{~mm}$ aminotriazole. Under these conditions, cell growth requires efficient GCN4- and TATA-dependent transcription. The altered specificity derivative of hTBP supports cell growth at lower aminotriazole concentrations, indicative of its ability to promote TATA-dependent Pol II transcription (Strubin and Struhl 1992). For each hybrid TBP, the portions derived from yTBP (solid bar) and hTBP (open bar) are indicated. Designations of the various hybrids are those used previously (Cormack et al. 1991). $\mathrm{H}^{*}$ is entirely hTBP except for amino acid 231 .

change at amino acid 231 of hTBP, resulting in a substitution of lysine for arginine (Fig. 2). Interestingly, the corresponding amino acid in YTBP (residue 133) is lysine, indicating that the $\mathrm{R} 231 \mathrm{~K}$ mutation results in the replacement of a human residue by its yeast counterpart. This amino acid is one of the eight basic residues that make up the TBP "basic repeat" (Horikoshi et al. 1989), and it is the only such basic residue that is not conserved between yeast and human.

When the $\mathrm{R} 231 \mathrm{~K}$ mutation is introduced into an otherwise wild-type hTBP, the resulting protein supports cell growth at $30^{\circ} \mathrm{C}$ (Fig. 3). Thus, we have identified one of the critical amino acid differences between yeast and human TBP that contributes to species specificity in vivo. However, strains containing the $\mathrm{R} 231 \mathrm{~K}$ derivative of hTBP grow more slowly than wild-type yeast strains at $30^{\circ} \mathrm{C}$, and they fail to grow at $37^{\circ} \mathrm{C}$. The temperaturesensitive phenotype could reflect thermolability of this TBP derivative or, more likely in our opinion, a general failure of slow-growing strains to thrive in more stringent conditions. The observation that the R231 derivative of hTBP is not fully functional in yeast is consistent with previous results (Cormack et al. 1991; Gill and Tjian 1991) that multiple differences between hTBP and yTBP are important for species specificity.

The failure of hTBP to support yeast cell growth does not correlate with a decrease in TATAdependent, Pol II transcription

Although results presented here (Fig. 1) and elsewhere 
(Kelleher et al. 1992; Strubin and Struhl 1992) indicate that hTBP supports basal, TATA-dependent transcription and is responsive to acidic activator proteins, the possibility remained that species specificity might be attributable to the relative inefficiency of hTBP in carrying out these functions. To address this issue, we utilized an assay for TBP function that is specific for TATA-dependent transcription in vivo (Strubin and Struhl 1992). In this assay TBP derivatives with altered DNA-binding specificity are examined for their ability to stimulate transcription from a HIS3 promoter that contains a mutated TATA element, TGTAAA. Wild-type yTBP is present in the cell to permit normal cell growth, but it functions very poorly at this promoter. Altered DNAbinding specificity versions of yTBP and hTBP (respectively termed yTBP ${ }^{\mathrm{m} 3}$ and $\mathrm{hTBP}^{\mathrm{m} 3}$ ) allow increased transcription of the TGTAAA-containing HIS3 promoter, with the YTBP derivative being more efficient (Strubin and Struhl 1992).

Altered specificity (i.e., m3) versions of hTBP, hTBP carrying the $\mathrm{R} 231 \mathrm{~K}$ mutation that allows hTBP to support cell growth, and hTBP/yTBP hybrid 17 were introduced into a strain carrying the TGTAAA-containing HIS3 promoter and tested for their ability to confer growth in the presence of $5 \mathrm{~mm}$ aminotriazole, a competitive inhibitor of the HIS3 gene product. Under these relatively stringent conditions, $\mathrm{yTBP}^{\mathrm{m} 3}$ supports growth, whereas hTBP ${ }^{\mathrm{m} 3}$ does not (Strubin and Struhl 1992). As shown in Figure 3, there was no correlation between the ability to support cell growth in the plasmid shuffle assay, and the ability of the corresponding $\mathrm{m} 3$ derivative to support transcription of TGTAAA-containing promoter. The strain carrying hybrid 17 is only marginally viable, yet hybrid $17^{\mathrm{m} 3}$ supports HIS3 transcription well. Conversely, hTBP carrying the $\mathrm{R} 231 \mathrm{~K}$ mutation supports cell growth reasonably well, yet its $\mathrm{m} 3$ version is no more functional for TATA-dependent transcription than $\mathrm{hTBP}^{\mathrm{m} 3}$ itself. Thus, the failure of hTBP to functionally replace yTBP in vivo cannot be explained by a general decrease in TATA-dependent, Pol II transcription.

As an independent test for Pol II function, we also examined whether hTBP could complement the growth defect associated with yTBPm3 ${ }^{3}$. This derivative is unable to support cell growth, presumably because altered TATA element recognition leads to a defect in Pol II transcription (Strubin and Struhl 1992). As shown in Figure 4, hTBP partially complements the growth defect of yTBP $^{m}$; a strain containing hTBP and yTBP ${ }^{\mathrm{m} 3}$ is viable, although it grows more slowly than a wild-type strain. Moreover, hybrid 17, which differs from hTBP by only 10 residues at the carboxyl terminus, almost completely complements the growth defect associated with yTBP ${ }^{\mathrm{m} 3}$. The relative abilities of hybrid 17 and hTBP to complement yTBP $^{\mathrm{m} 3}$ correlate well with their relative efficiencies in promoting TATA-dependent Pol II transcription (Fig. 3).

\section{hTBP is defective for Pol III transcription}

In a detailed mutational analysis of yTBP, we isolated a

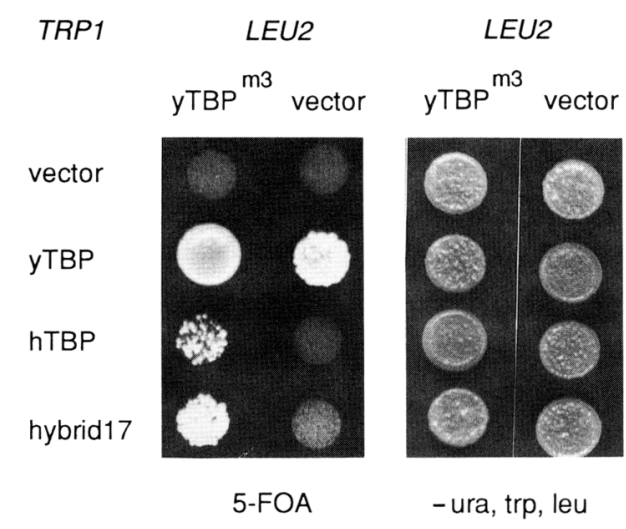

Figure 4. hTBP complements the growth defect associated with the yTBP derivative $(\mathrm{m} 3)$ with altered TATA element specificity. Strains containing the indicated TRP1 or LEU2 TBP plasmids (or control vectors) were tested for their ability to grow in the presence of 5-FOA after plasmid shuffling or in selective minimal medium (i.e., lacking uracil, tryptophan, and leucine) that requires the maintenance of all plasmids.

large number of temperature-sensitive derivatives that are specifically defective in Pol III transcription at the restrictive temperature (Cormack and Struhl 1993). These mutations define a TBP surface that may interact with Tds4/Brfl/Pcf4, a component of the Pol III transcription factor TFIIIB (Buratowski and Zhou 1992; Colbert and Hahn 1992; Lopez-De-Leon et al. 1992), because overproduction of Tds4 suppresses the temperature-sensitive phenotype of Pol III-specific TBP mutations (Cormack and Struhl 1993). We examined hTBP for its ability to complement the temperature-sensitive phenotype of two different Pol III-specific mutations (F133S and Y231L). As shown in Figure 5, strains containing hTBP and either of the Pol III-specific mutations fail to grow at $37^{\circ} \mathrm{C}$. As a positive control for this assay, we examined the A135T derivative of y TBP, which shows a preferential defect in Pol I and Pol II transcription (Cormack and Struhl 1993). Strains containing A135T and either of the Pol III-specific derivatives grow at $37^{\circ} \mathrm{C}$, although somewhat slower than a strain containing wild-type yTBP. Thus, by this genetic assay, hTBP is unable to support sufficient Pol III transcription for cell growth.

\section{hTBP functions poorly at Pol I, Pol III, and TATA-less Pol II promoters}

The above experiments demonstrate that the failure of hTBP derivatives to support cell growth does not correlate with the ability to support TATA-dependent Pol II transcription but may be related to a defect in Pol III transcription. To assess directly the transcriptional activity of hTBP at different classes of yeast promoters, we exploited two temperature-sensitive alleles of yTBP (T111I and S136N) that are defective for Pol I, Pol II, and Pol III transcription at the restrictive temperature (Cormack and Struhl 1992). By expressing hTBP in these mutant strains and eliminating yTBP function by shifting 


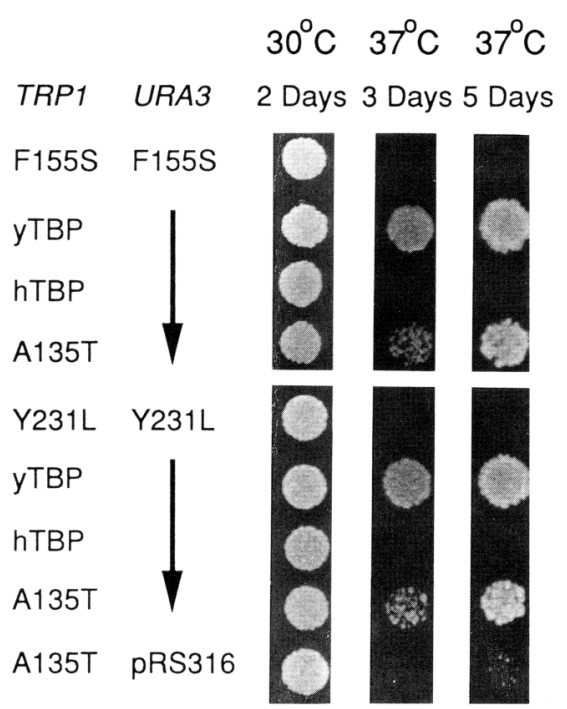

Figure 5. hTBP does not not complement TBP derivatives with Pol III-specific defects. Strains containing either of two temperature-sensitive, Pol III-specific derivatives of yTBP (F155S or Y231L; Cormack and Struhl 1993) on URA3 centromeric plasmids and the indicated TBP derivatives on TRP1 centromeric plasmids were tested for their ability to grow at $37^{\circ} \mathrm{C}$. The A 135T derivative of yTBP, which preferentially affects Pol I and Pol II transcription (Cormack and Struhl 1993), complements the Pol III-defective TBPs, but hTBP does not.

cells to the restrictive temperature, we could assess whether hTBP was able to function at promoters utilized by all three nuclear RNA polymerases. Specifically, at various times after the temperature shift, we quantitated mRNA levels for TATA-dependent (HIS3 $+13, D E D 1)$ and TATA-less (HIS3 +1 , TRP3) Pol II promoters, as well as RNA levels corresponding to the intron of the Pol III-transcribed isoleucine tRNA and the junction between the mature $25 \mathrm{~S}$ rRNA and the 3 '-nontranscribed spacer of the Pol I-transcribed ribosomal precursor RNA. Because the half-lives of these RNA species are short, the signal at any given time reflects transcriptional initiation, not accumulated RNA levels (Cormack and Struhl 1992).

As shown previously, T111I (Fig. 6) and S136N (data not shown) strains show a rapid decrease in all of these RNA species after the shift to the restrictive temperature, reflecting the common role of TBP in Pol I, Pol II, and Pol III transcription. In the wild-type strain, the levels of these RNA species are not affected by shift to $37^{\circ} \mathrm{C}$ (Fig. 6), and coexpression of hTBP has no effect (data not shown). In accord with results presented here and elsewhere, coexpression of hTBP in T111I strain restores transcription from TATA-containing Pol II promoters $(D E D 1$ and $H I S 3+13)$ at the restrictive temperature. In contrast, transcription from TATA-less promoters (TRP3 and HIS $3+1$ ) declined as rapidly after temperature shift as in the T111I strain not expressing hTBP. Levels of tRNA and rRNA also fell equally rapidly in the T111I strains that did or did not express hTBP. Identical results for all these promoters were observed in $\$ 136 \mathrm{~N}$ strains shifted to the restrictive temperature (data not shown). Thus, in yeast cells, hTBP is functional for Pol II transcription dependent on a consensus TATA element but it is unable to support efficient transcription from Pol I, Pol III, and TATA-less Pol II promoters.

\section{Discussion}

We present five independent lines of evidence that the failure of hTBP to support yeast cell growth is not attributable to a general failure of Pol II transcription but, rather, to its inability to support Pol I, Pol III, and TATAless Pol II transcription. First, chimeric hTBP-yTBP molecules that display a wide range in their ability to support yeast cell growth do not show corresponding defects in transcription from the Pol II promoters HIS 3 and $D E D 1$ (Fig. 1). Ironically, those chimeric proteins least able to support cell growth show the highest levels of HIS3 +13 transcription. Although this effect is indirect, being caused by induction of GCN4 as a consequence of

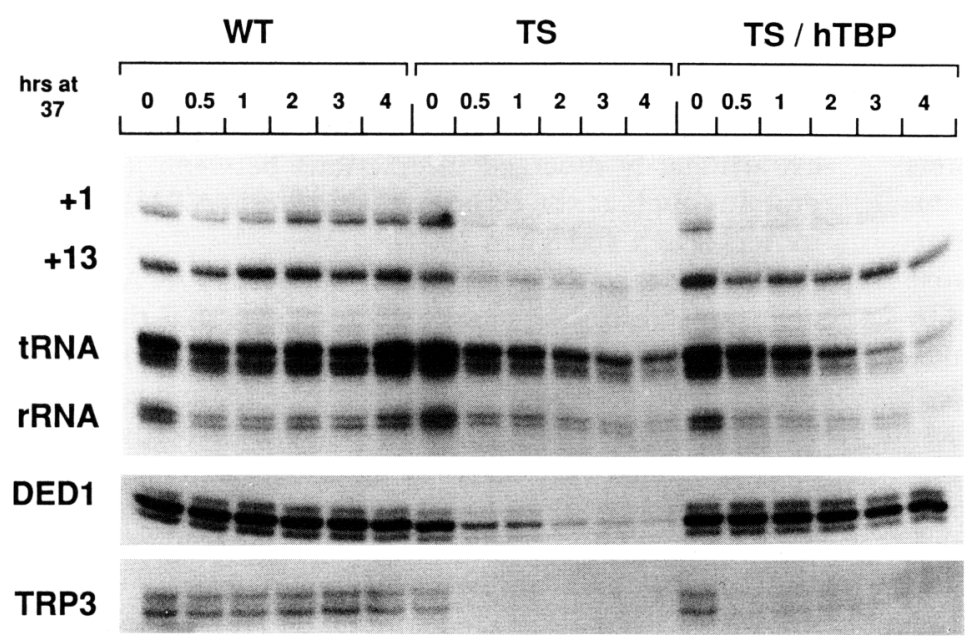

Figure 6. Transcriptional analysis. RNA levels of the indicated genes in strains containing wild-type yTBP, a temperature-sensitive derivative of YTBP (T111I), or the combination of T111I and hTBP at various times in hours) after shifting the cultures to $37^{\circ} \mathrm{C}$. 
poor cell growth, it emphasizes the fact that even the most functionally compromised chimeric TBPs carry out Pol II transcription and respond to acidic activators.

Second, hTBP partially complements the growth defect associated with the YTBP derivative that has altered TATA element recognition properties (Fig. 4). This observation suggests that hTBP carries out sufficient Pol II function to support viability. Consistent with this view, cell viability is not correlated with the relative efficiency at supporting TATA-dependent transcription (Fig. 3).

Third, hTBP does not complement the Pol III-specific defect of two yTBP derivatives, indicating that it is defective for Pol III transcription (Fig. 5). As discussed below, it is unlikely that hTBPs lack of function in this assay reflects instability/unfolding of the protein at $37^{\circ} \mathrm{C}$. This observation suggests that the defect in Pol III transcription could account for the inability of hTBP to support yeast cell growth.

Fourth, we isolated three independent intragenic suppressors in hTBP that permit yeast cell growth but do not increase the efficiency of TATA-dependent Pol II transcription (Fig. 3). Strikingly, all three derivatives contain the same mutation, which replaces arginine 231 of hTBP with lysine, the corresponding yeast amino acid. The corresponding position in yTBP $(\mathrm{K} 133)$, which is located at the amino terminus of helix $\mathrm{Hl}$ (Nikolov et al. 1992), has been implicated in Pol III transcription. Overexpression of Tds4/Brf1/Pcf4, the $70-\mathrm{kD}$ subunit of Pol III factor TFIIIB, suppresses the double mutant K133L, K138L, consistent with a role for K133 in Pol III transcription (Buratowski and Zhou 1992). In a separate study, we isolated a large number of temperature-sensitive mutations defective in Pol III transcription but functional for Pol II transcription (Cormack and Struhl 1993). Among these were two substitutions at K133, again implicating that residue in Pol III transcription. The fact that substitution of lysine for arginine at this position permits cell viability suggests that the major defect of hTBP in yeast cells is in Pol III, not Pol II transcription.

Finally, by shifting cells that are coexpressing hTBP and a temperature-sensitive yTBP to the restrictive temperature, we directly assayed the transcriptional activity of hTBP at various classes of promoters (Fig. 6). Interestingly, and consistent with the other studies, transcription from the two TATA-element containing promoters (DED1, HIS3 + 13) occurred at a level comparable to that in the wild-type strain. In contrast, transcripts from the tRNA, rRNA, and two TATA-less Pol II promoters (TRP3, HIS3 + 1) are not observed at the restrictive temperature. The effects at the Pol II promoters are not attributable to growth-related induction of GCN4 because TRP3 and the HIS3 + 13 transcripts are both activated by GCN4 yet are differentially affected under these conditions. Thus, at $37^{\circ} \mathrm{C}$, hTBP supports TATA-dependent Pol II transcription but it is defective for Pol I, Pol III, and TATA-less Pol II transcription.

The fact that hTBP supports efficient TATA-dependent Pol II transcription argues strongly that the other functional defects are not attributable to instability or misfolding of hTBP at the elevated temperature neces- sary to carry out this experiment. hTBP itself is not inherently temperature sensitive for these specific functions, because human cells grow normally at $37^{\circ} \mathrm{C}$. Thus, we conclude that the functional defects observed at $37^{\circ} \mathrm{C}$ reflect species-specific incompatibilies between hTBP and yeast transcription factors. Although we cannot be sure that the transcriptional defects seen at $37^{\circ} \mathrm{C}$ (Fig. 6) are the same as those responsible for inviability of hTBP at $30^{\circ} \mathrm{C}$, we think it highly likely. Finally, we note that although our results clearly show functional defects of hTBP at particular classes of promoters, it remains possible that hTBP might not behave identically on all promoters of a given class.

\section{Molecular implications}

Transcription by all three nuclear RNA polymerases requires the presence of TBP at the promoter region (for review, see Sharp 1992; Hernandez 1993; Struhl 1994). In the case of Pol II promoters containing TATA elements, TBP can bind directly to the promoter. Moreover, the TBP-TATA element interaction is the first step in assembling an active Pol II transcription complex, and the TATA element is likely to be a critical DNA sequence determinant of the promoter. For other promoters, recruitment of TBP to the promoter is unlikely to occur by DNA sequence recognition and, hence, is presumably achieved by protein-protein interactions. In mammalian cells, TBP interacts with associated proteins (TAFs) to form SL1, TFIID, and TFIIIB complexes that are specific for Pol I, Pol II, and Pol III transcription. In yeast, equivalent complexes probably exist, although only TFIIIB has been identified.

Our results indicate that in yeast cells hTBP is defective at promoters in which recruitment requires proteinprotein interactions. For Pol I and Pol III transcription, the simplest model to account for the functional differences between yTBP and hTBP invokes species-specific interactions between TBP and TAFs that are necessary for the formation/activity of the SL1 and TFIIIB complexes. Given the importance of lysine 133 of yTBP in species specificity, and the fact that overexpression of Tds4 suppresses the Pol III-specific defect caused by mutations at this residue of yTBP /Cormack and Struhl 1993), it is plausible that the putative interaction between TBP and Tds4 is incompatible across species. For Pol II transcription, functional differences might reflect evolutionarily diverged interactions between TBP and proteins such as the tethering factor for TATA-less promoters (Pugh and Tjian 1991).

The fact that hTBP efficiently supports TATA-dependent transcription in yeast cells indicates that the interactions of TBP with basic components of the Pol II transcription machinery (e.g., TFIIB and TFIIA) and with proteins involved in the response to acidic activators must be functionally conserved. In addition, our results indicate that any TBP-TAF interactions important to TATA-dependent Pol II transcription must be functionally conserved across species. However, it is not yet known whether TAFs are required for TATA-dependent, 
Pol II transcription in yeast. In this regard, free TBP has never been detected in mammalian cell extracts, but it is present in considerable amounts in yeast cell extracts (Buratowski et al. 1988; Cavallini et al. 1988). Moreover, TBP appears to be sufficient for activated transcription in a yeast in vitro transcription system (Kelleher et al. 1992), whereas Pol II-specific TAFs are clearly required for the response to activator proteins in mammalian in vitro transcription systems (Dynlacht et al. 1991; Tanese et al. 1991; Takada et al. 1992; Zhou et al. 1992; Wang and Tjian 1994). The role(s) of TAFs in yeast Pol II transcription and the molecular details of species-specific interactions between TBP and TAFs remain to be clarified.

\section{Materials and methods}

Strains and plasmids

Strain BY $\Delta 2$, which is deleted for the chromosomal TBP locus, and derivatives carrying the temperature sensitive (ts) yeast/ human hybrid TBP alleles on centromeric TRP1 plasmids have been described previously (Cormack et al. 1991; Cormack and Struhl 1992, 1993). When necessary, TBP derivatives were recloned into URA3 or LEU2 centromeric plasmids. The hTBP and the hybrid $\mathrm{hTBP} / \mathrm{yTBP}$ expression constructs consist of a precise fusion of the yTBP promoter and 3 '-untranslated region to the coding region of hTBP (Cormack et al. 1991). The hTBP derivative containing the $\mathrm{R} 231 \mathrm{~K}$ mutation was generated by substituting the PstI-XbaI fragment from the mutated hybrid 17 molecule into hybrid 11 (Cormack et al. 1991), generating an entirely human derived hTBP except for the single amino acid change. The altered specificity mutations were introduced into this R $231 \mathrm{~K}$ hTBP derivative by substitution of the PstI-XbaI fragment into the altered specificity hTBP molecule generated previously (Strubin and Struhl 1992). The three mutations that confer altered specificity were introduced into hybrid 17 by polymerase chain reaction using hybrid molecule 17 as template and oligonucleotides $\mathrm{hBcll}$ and $\mathrm{hBcl} 2$ as primers $(\mathrm{hBcll}$ : CCGCTGATCAAACCCAGAATTACTCTCGTTATTTTTG; hBcl2: GCGCTGATCATTCTGTAGAATAAACCAGG). After cleavage with $B c l I$, the two amplified products were ligated together, and the resulting product cleaved with $\mathrm{XbaI}$ and $\mathrm{BamHI}$ before being inserted into hybrid molecule 12 (Cormack et al. 1991 ) in place of the yeast sequence.

\section{$T B P$ phenotypic analysis}

To analyze the various TBPs for their ability to support GCN4and TATA-dependent activation, altered specificity derivatives were introduced into a strain containing the his 3 allele that contains a GCN4-binding site upstream of the TGTAAA TATA element as described previously (Strubin and Struhl 1992). Transformants were tested for growth by spotting $\sim 10^{4}$ cells onto SD media lacking histidine and supplemented with $5 \mathrm{~mm}$ aminotriazole, a competitive inhibitor of the HIS3 gene product. The level of resistance to aminotriazole is directly related to the level of HIS3 transcription (Hill et al. 1986; Chen and Struhl 1988). Under these stringent assay conditions, yTBP supports cell growth, whereas hTBP does not; however, hTBP does support GCN4- and TATA-dependent activation as indicated by its ability to support cell growth at lower aminotriazole concentrations (Strubin and Struhl 1992). For the plasmid shuffle assay, the relevant TBP derivatives, carried on a TRP1 centromeric vector, were introduced into BY $\Delta 2$ whose only copy of TBP is carried on a centromeric URA3 vector. The transfor- mants were grown on media lacking uracil and tryptophan to select for the TRP 1 and $U R A 3$ vectors, and $\sim 10^{5}$ cells were then plated on SD media containing 5-FOA. The complementation assay for Pol III function was carried out by introducing TBP derivatives into strains containing the F155S and Y231 alleles (Cormack and Struhl 1993) and testing for growth at $37^{\circ} \mathrm{C}$.

\section{RNA analysis}

For the experiments in Figure 1, the various strains were grown in YPD media to early log phase $\left(A_{550}=0.5\right)$, collected by centrifugation, and frozen. For the temperature-shift experiments (Fig. 6), cells were grown in YPD medium to early exponential phase at $30^{\circ} \mathrm{C}$, shifted to $37^{\circ} \mathrm{C}$ for $15 \mathrm{~min}$, and returned to $30^{\circ} \mathrm{C}$ for $1 \mathrm{hr}$. After this pre-heat shock, the cultures were shifted back to $37^{\circ} \mathrm{C}$ and aliquots taken at the appropriate times. As described previously (Cormack and Struhl 1992), the pre-heat shock induces the heat shock proteins and prevents the transient $(\sim 30 \mathrm{~min})$ decrease in all transcripts that occurs under these mild heat shock conditions (Nicolet and Craig 1991). For each time point the cells were collected rapidly by centrifugation and flash frozen in liquid nitrogen.

Total RNA was prepared from each sample of cells and quantitated by absorbance at $260 \mathrm{nM}$ and by staining with ethidium bromide after separation by agarose gel electrophoresis. Ten micrograms of RNA from each sample was hybridized to completion with a 100 -fold excess of the appropriate ${ }^{32} \mathrm{P}$-labeled oligonucleotides and treated with SI nuclease as described previously (Cormack and Struhl 1992).

\section{Acknowledgments}

We thank Rachel Green for a critical reading of the manuscript. This work was supported by postdoctoral fellowships from the Swiss National Science Foundation (M.S.) and the American Cancer Society (L.A.S.), by a predoctoral fellowship from the Howard Hughes Medical Institute (B.P.C.), and by research grants to K.S. from the National Institutes of Health (GM30186) and the Lucille Markey Trust.

The publication costs of this article were defrayed in part by payment of page charges. This article must therefore be hereby marked "advertisement" in accordance with 18 USC section 1734 solely to indicate this fact.

\section{References}

Berkenstam, A., M.M. Ruiz, D. Barettino, M. Horikoshi, and H.G. Stunnenberg. 1992. Cooperativity in transactivation between retinoic acid receptor and TFIID requires an activity analogous to E1A. Cell 69: 401-412.

Bossier, P., L. Fernandes, D. Rocha, and C. Rodrigues-Pousada. 1993. Overexpression of $Y A P 2$, coding for a new yAP protein, and YAP1 in Saccharomyces cerevisiae alleviates growth inhibition caused by 1,10-phenanthroline. J. Biol. Chem. 268: 23640-23645.

Buratowski, S. and H. Zhou. 1992. A suppressor of TBP mutations encodes an RNA polymerase III transcription factor with homology to TFIIB. Cell 71: 221-230.

Buratowski, S., S. Hahn, P.A. Sharp, and L. Guarente. 1988. Function of a yeast TATA element-binding protein in a mammalian transcription system. Nature 334: 37-42.

Cavallini, B., J. Huet, J.L. Plassat, A. Sentenac, J.-M. Egly, and P. Chambon. 1988. A yeast activity can substitute for the HeLa TATA box factor. Nature 334: 77-80.

Chen, W. and K. Struhl. 1988. Saturation mutagenesis of a yeast 
his3 TATA element: Genetic evidence for a specific TATAbinding protein. Proc. Nat1. Acad. Sci. 85: 2691-2695.

Colbert, T. and S. Hahn. 1992. Isolation of a TFIIB related factor involved in RNA polymerase III transcription. Genes \& Dev. 6: $1940-1949$.

Comai, L., N. Tanese, and R. Tjian. 1992. The TATA-binding protein and associated factors are integral components of the RNA polymerase I transcription factor, SLl. Cell 68: 965976.

Cormack, B.P. and K. Struhl. 1992. The TATA-binding protein is required for transcription by all three nuclear RNA polymerases in yeast cells. Cell 69: 685-696.

1993. Regional codon randomization: Defining a TATAbinding protein surface required for RNA polymerase III transcription. Science 262: 244-248.

Cormack, B.P., M. Strubin, A.S. Ponticelli, and K. Struhl. 1991. Functional differences between yeast and human TFIID are localized to the highly conserved region. Cell 65: 341-348.

Dynlacht, B.D., T. Hoey, and R. Tijan. 1991. Isolation of coactivators associated with the TATA-binding protein that mediate transcriptional activation. Cell 66: 563-576.

Flanagan, P.M., R.J.I. Kelleher, W.J. Feaver, N.F. Lue, J.W. LaPointe, and R.D. Kornberg. 1990. Resolution of factors required for the initiation of transcription by yeast RNA polymerase II. I. Biol. Chem. 265: 11105-11107.

Gill, G. and R. Tjian. 1991. A highly conserved domain of TFIID displays species specificity in vivo. Cell 65: 333-340.

Hernandez, N. 1993. TBP, a universal eukaryotic transcription factor? Genes \& Dev. 7: 1291-1308.

Hill, D.E., I.A. Hope, J.P. Macke, and K. Struhl. 1986. Saturation mutagenesis of the yeast HIS 3 regulatory site: Requirements for transcriptional induction and for binding by GCN4 activator protein. Science 234: $451-457$.

Hinnebusch, A.G. 1984. Evidence for translational regulation of the activator of general amino acid control in yeast. Proc. Natl. Acad. Sci. 81: 6442-6446.

Horikoshi, M., C.K. Wang, H. Fujii, J.A. Cromlish, P.A. Weil, and R.G. Roeder. 1989. Cloning and structure of a yeast gene encoding a general transcription initiation factor TFIID that binds to the TATA box. Nature 341: 299-303.

Horikoshi, M., T. Yamamoto, Y. Ohkuma, P.A. Weil, and R.G. Roeder. 1990. Analysis of structure-function relationships of yeast TATA box binding factor TFID. Cell 61: 1171-1178.

Kassavetis, G.A., C.A.P. Joazeiro, M. Pisano, E.P. Geiduschek, T. Colbert, S. Hahn, and J.A. Blanco. 1992. The role of the TATA-binding protein in the assembly and function of the multisubunit yeast RNA polymerase transcription factor, TFIIIB. Cell 71: 1055-1064.

Keaveney, M., A. Berkenstam, M. Feigenbutz, G. Vriend, and H.G. Stunnenberg. 1993. Residues in the TATA-binding protein required to mediate a transcriptional response to retinoic acid in EC cells. Nature 365: 562-566.

Kelleher, R.J.I., P.M. Flanagan, D.I. Chasman, A.S. Ponticello, K. Struhl, and R.D. Kornberg. 1992. Yeast and human TFIIDs are interchangeable for the response to acidic transcriptional activators in vitro. Genes \& Dev. 6: 296-303.

Kim, J.L., D.B. Nikolov, and S.K. Burley. 1993. Co-crystal structure of TBP recognizing the minor groove of a TATA element. Nature 365: 520-527.

Kim, Y., J.H. Geiger, S. Hahn, and P.B. Sigler. 1993. Crystal structure of a yeast TBP-TATA box complex. Nature 365: 512-520.

Lobo, S.M., M. Tanaka, M.L. Sullivan, and N. Hernandez. 1992. A TBP complex essential for transcription from TATA-less but not TATA-containing RNA polymerase III promoters is part of the TFIIIB fraction. Cell 71: 1029-1040.
Lopez-De-Leon, A., M. Librizzi, K. Puglia, and I.M. Willis. 1992. PCF4 encodes an RNA polymerase III transcription factor with homology with TFIIB. Cell 71: 211-220.

Mosch, H.U., B. Scheier, R. Lahti, P. Mantsala, and G.H. Braus. 1991. Transcriptional activation of yeast nucleotide biosynthetic gene ADE4 by GCN4. J. Biol. Chem. 266: 2045320456.

Moye-Rowley, W.S., K.D. Harshman, and C.S. Parker. 1989. Yeast YAP1 encodes a novel form of the iun family of transcriptional activator proteins. Genes \& Dev. 3: 283-292.

Nicolet, C.M. and E.A. Craig. 1991. Inducing and assaying heat shock response in Saccharomyces cerevisiae. Methods Enzymol. 194: 710-717.

Nikolov, D.B., S.-H. Hu, J. Lin, A. Gasch, A. Hoffman, M. Horikoshi, N.-H. Chua, R.G. Roeder, and S.K. Burley. 1992. Crystal structure of TFIID TATA-box binding protein. Nature 360: $40-46$.

Peterson, M.G., N. Tanese, F. Pugh, and R. Tjian. 1990. Functional domains and upstream activation properties of cloned human TATA binding proteins. Science 248: 1625-1630.

Poon, D., S. Schroeder, C.K. Wang, T. Yamamoto, M. Horikoshi, R.G. Roeder, and P.A. Weil. 1991. The conserved carboxyterminal domain of Saccharomyces cerevisiae TFIID is sufficient to support normal cell growth. Mol. Cell. Biol. 11: 4809-4821.

Pugh, B.F. and R. Tjian. 1990. Mechanism of transcriptional activation by Sp1: evidence for coactivators. Cell 61: 11871197.

- 1991. Transcription from a TATA-less promoter requires a multisubunit TFIID complex. Genes \& Dev. 5: 1935-1945.

Rolfes, R.J. and A.G. Hinnebusch. 1993. Translation of the yeast transcriptional activator GCN4 is stimulated by purine lim. itation: Implications for activation of the protein kinase GCN2. Mol. Cell. Biol. 13: 5099-5111.

Schnell, N., B. Krems, and K.D. Entian. 1992. The PAR1 (YAP1/ SNQ3) gene of Saccharomyces cerevisiae, a c-jun homologue, is involved in oxygen metabolism. Curr. Genet. 21: 269-273.

Schultz, M.C., R.H. Reeder, and S. Hahn. 1992. Variants of the TATA-binding protein can distinguish subsets of RNA polymerase I, II, and III promoters. Cell 69: 697-702.

Sharp, P.A. 1992. TATA-binding protein is a classless factor. Cell 68: 819-821.

Strubin, M. and K. Struhl. 1992. Yeast TFIID with altered DNAbinding specificity for TATA elements. Cell 68: 721-730.

Struhl, K. 1986. Constitutive and inducible Saccharomyces cerevisiae promoters: Evidence for two distinct molecular mechanisms. Mol. Cell. Biol. 6: 3847-3853.

- 1994. Duality of TBP, the universal transcription factor. Science 263: 1103-1104.

Taggart, A.K.P., T.S. Fisher, and B.F. Pugh. 1992. The TATAbinding protein and associated factors are components of Pol III transcription factor TFIIIB. Cell 71: 1015-1028.

Takada, R., Y. Nakatani, A. Hoffman, T. Kokubo, S. Hasegawa, R.G. Roeder, and M. Horikoshi. 1992. Identification of human TFIID components and direct interaction between a $250 \mathrm{kDa}$ polypeptide and the TATA-box binding protein (TFIID tau). Proc. Natl. Acad. Sci. 89: 11809-11813.

Tanese, N., B.F. Pugh, and R. Tjian. 1991. Coactivators for a proline-rich activator purified from the multisubunit human TFIID complex. Genes \& Dev. 5: 2212-2224.

Thireos, G., M.D. Penn, and H. Greer. 1984. 5' Untranslated sequences are required for the translational control of a yeast regulatory gene. Proc. Natl. Acad. Sci. 81: 5096-5100.

Wang, E.H. and R. Tiian. 1994. Promoter-selective transcrip- 
tional defect in cell cycle mutant ts 13 rescued by hTAF 250 . Science 263: 811-814.

White, R.J., S.P. Jackson, and P.W.J. Rigby. 1992. A role for the TATA-box-binding protein component of the transcription factor TFIID complex as a general RNA polymerase III transcription factor. Proc. Natl. Acad. Sci. 89: 1949-1953.

Wobbe, C.R. and K. Struhl. 1990. Yeast and human TATA-binding proteins have nearly identical DNA sequence requirements for transcription in vitro. Mol. Cell. Biol. 10: 38593867.

Zhou, Q., M.C. Schmidt, and A.J. Berk. 1991. Requirement for acidic amino acid residues immediately $\mathrm{N}$-terminal to the conserved domain of Saccharomyces cerevisiae TFIID. EMBO I. 10: 1843-1852.

Zhou, Q., P.M. Lieberman, T.G. Boyer, and A.J. Berk. 1992. Holo TFIID supports transcriptional stimulation by diverse activators and from a TATA-less promoter. Genes \& Dev. 6: 1964-1974. 


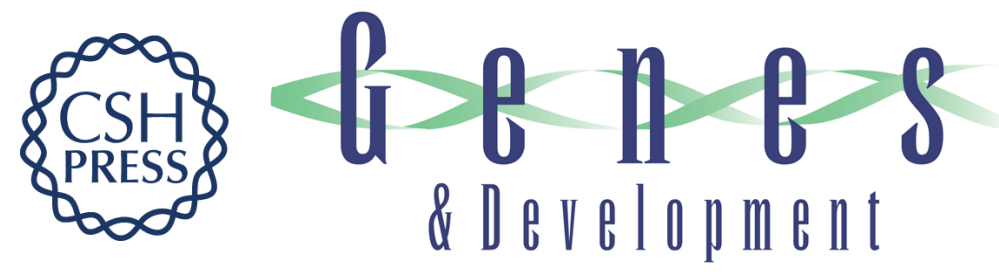

\section{Conserved and nonconserved functions of the yeast and human TATA-binding proteins.}

B P Cormack, M Strubin, L A Stargell, et al.

Genes Dev. 1994, 8:

Access the most recent version at doi:10.1101/gad.8.11.1335

References This article cites 50 articles, 24 of which can be accessed free at:

http://genesdev.cshlp.org/content/8/11/1335.full.html\#ref-list-1

License

Email Alerting Receive free email alerts when new articles cite this article - sign up in the box at the top Service right corner of the article or click here.

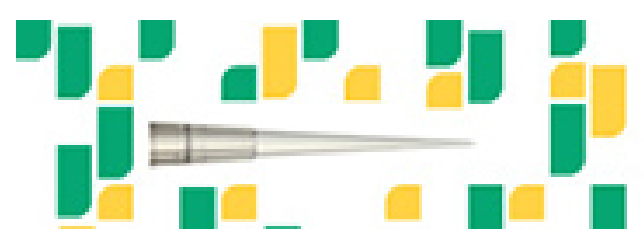

Focused on your science.

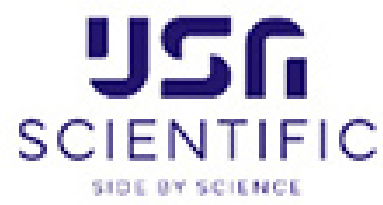

Copyright (c) Cold Spring Harbor Laboratory Press 